\title{
ANÁLISIS COMPARATIVO DE LA COMPLEJIDAD COGNITIVA DE LA GESTIÓN DE PROYECTO EN EL DESARROLLO DE SOFTWARE EN LA ISO/IEC 29110-5-1-2 Y LA GUÍA DE LOS FUNDAMENTOS PARA LA DIRECCIÓN DE PROYECTOS DEL PMI
}

COMPARATIVE ANALYSIS OF THE COGNITIVE COMPLEXITY OF THE PROJECT MANAGEMENT IN THE SOFTWARE DEVELOPMENT IN ISO/IEC 29110-5-1-2 AND THE GUIDE OF THE FUNDAMENTALS FOR THE MANAGEMENT OF PMI PROJECTS

Edinson Damian ${ }^{1}$

edacosta@unprg.edu.pe

Abraham Dávila²

abraham.davila@pucp.edu.pe

${ }^{1}$ Escuela de Posgrado - Universidad Nacional Pedro Ruiz Gallo Av. Huamachuco 1130, Lambayeque

Lambayeque, Perú

${ }^{2}$ Departamenteo de Ingeniería - Pontificia Universidad Católica del Perú Av.Universitaria 1801, Urb. Pando, San Miguel

Lima, Perú 


\title{
RESUMEN
}

El desarrollo de marcos de trabajo de procesos ha contribuido en la consolidación de la industria de software. Sin embargo, la dificultad de adopción de esos marcos de trabajo ha llevado al desarrollo de métricas para evaluar, entre otros aspectos, su complejidad. En este contexto, se ha señalado que el proceso de gestión de proyectos descrito en la ISO/IEC 29110-5-1-2 es menos complejo que el descrito en la Guía de los fundamentos para la dirección de proyecto del PMI. Para comprobar dicha afirmación se realizó un análisis comparativo de la complejidad cognitiva. La medición se aplicó a los entregables, actividades y proceso. A partir de lo obtenido, la descripción del proceso de gestión de proyectos de la ISO/IEC 29110-5-1-2 es menos compleja a nivel cognitivo que lo descrito en la Guía del PMI.

\section{PALABRAS CLAVE}

Complejidad Cognitiva; Dirección de Proyectos; ISO/IEC 29110.

\begin{abstract}
The development of process frameworks has contributed to the consolidation of the software industry. However, the difficulty of adopting these frameworks has led to the development of metrics to evaluate, among other aspects, their complexity. In this context, it has been pointed out that the project management process described in ISO/IEC 29110-5-1-2 is less complex than the one described in the Project Management Foundations Guide of PMI. To verify this statement, a comparative analysis of the cognitive complexity was performed. The measurement was applied to the deliverables, activities and process. From the results obtained, that the description of the project management process of ISO/IEC 29110-5-1-2 is less complex at a cognitive level than that described in the PMI Guide.
\end{abstract}

\section{KEYWORDS}

Cognitive complexity; Project management; ISO/IEC 29110. 


\section{INTRODUCCIÓN}

Un aspecto relevante en el contexto de la industria de software y la adopción de modelos de proceso software es saber si la complejidad de un modelo es adecuada para su adopción en la organización. Sí bien se han desarrollado estudios sobre la medición de proceso software como (Mitani et al., 2007), (Oliveira, Oliveira, \& Belchior, 2006), (Slavek, Lukić, \& Köhler, 2012), y (Ram, Rodriguez, \& Oivo, 2018), ninguno de ellos evalúa la complejidad del modelo como tal. En contraste se tiene que: (i) en (Rossi \& Brinkkemper, 1996) se introduce el concepto de complejidad cognitiva como una medida del grado de complejidad conceptual en función de los factores de complejidad; (ii) en (Perepletchikov, Ryan, \& Tari, 2013) se propone un modelo jerárquico de la complejidad cognitiva de productos de trabajo, tareas y metodología; y (iii) en (García, Piattini, Ruiz, Canfora, \& Visaggio, 2006), se propone métricas relacionadas al proceso software y un marco para el modelado y evaluación de proceso software. En ese contexto, se reconoce que una organización que logra determinar la complejidad cognitiva de un modelo o metodología, podrá: estimar con mayor fiabilidad el esfuerzo para su implementación o tomar decisiones en el caso de una selección entre múltiples opciones.

De otro lado, la publicación de la ISO/IEC 29110 en el 2011 (ISO/IEC, 2016), representa un hito importante en la industria de software pues está pensada para las pequeñas organizaciones (empresas, áreas, unidades organizacionales, entre otras). Una de las características de esta Norma es que presenta dos procesos (Implementación de Software y Gestión de Proyecto) que son "más ligeros" y que se afirma más adecuado al tamaño de la organización (ISO/IEC, 2011). En particular, para el caso del proceso definido de Gestión de Proyecto, también se dice que es más ligero que una implementación completa de la Guía de los fundamentos para la dirección de proyectos del PMI (referida en la industria como PMBOK de Project Management Body of Knowledge) (PMI, 2017). Sin embargo, en la revisión bibliográfica no se ha encontrado un análisis comparativo de la ISO/IEC 29110-5-1-2 con alguna implementación completa del PMBOK desde la perspectiva de la complejidad cognitiva.

En este artículo se analiza de manera comparada los modelos de gestión de proyectos (en el contexto de la industria de software) de la ISO/IEC 29110-5-2 y una implementación basada en el PMBOK a partir de la determinación de las métricas de complejidad cognitiva. En lo que sigue, el artículo se organiza de la siguiente manera: en la Sección 2, se presenta un marco de referencia; en la Sección 3, se presentan las métricas construidas para realizar esta evaluación; en la Sección 4, se muestran los resultados de las mediciones y el análisis correspondiente; y en la Sección 5, presenta la discusión final y trabajo futuro.

\section{MARCO DE REFERENCIA}

El presente estudio se ha realizado tomando como base el concepto y métricas de complejidad cognitiva adaptados en su aplicación a procesos de la gestión de proyectos. A continuación se presentan: la complejidad cognitiva, métricas asociadas y la gestión de proyectos. 


\subsection{Complejidad Cognitiva}

En (Perepletchikov et al., 2013) se presenta un modelo de calidad para metodologías de desarrollo de software, como se observa en la Figura 1, organizado en 3 perspectivas, 7 características y 22 sub-características. El Modelo define (descompone) la calidad en 3 perspectivas, siendo relevante para nuestro estudio el relacionado al artefacto estructurado. Dicha perspectiva contiene una característica de comprensibilidad y 3 sub-características de complejidad cognitiva, con sus métricas asociadas. Las sub características de la complejidad cognitiva, siguiendo la notación de los autores, son: (i) S.C.1.1. Complejidad cognitiva del producto de trabajo que presenta 6 métricas; (ii) S.C.1.2 Complejidad cognitiva de las tareas que presenta 4 métricas; y (iii) S.C.1.3 Complejidad cognitiva de la metodología que presenta 14 métricas. De las métricas establecidas en las sub características de la perspectiva de artefacto estructurado (Perepletchikov et al., 2013), el criterio que se ha tomado como referencia es considerar solo aquellas que tienen relación directa con la construcción de un valor único final de complejidad cognitiva como se observa en la Tabla 1. En particular se puede identificar que M6 de la S.C.1.1 está en función de M1, M2 y M3 de la misma S.C.1.1; esta forma de agregar las métricas como distancia euclidiana es definida por los autores a partir del trabajo de Rossi y Brinkkemper, que se presenta en la Sección 2.2.

\subsection{Métricas de Rossi y Brinkkemper}

En (Rossi \& Brinkkemper, 1996) se presenta un conjunto de métricas para el evaluar la complejidad de técnicas y métodos para el desarrollo de sistemas en función de objeto, propiedad y relación. A partir de dicha definición se generan tres métricas como factores de complejidad individual (Rossi \& Brinkkemper, 1996) que permiten determinar la complejidad conceptual (ver Tabla 2). Para llegar a dichas métricas se establece que los elementos: Objeto, Propiedad y Relación-rol se conceptualizan como un espacio tridimensional y la medición como un punto en cada eje correspondiente a cada elemento. Por tal razón, la métrica propuesta por los autores se determinar como una distancia (euclidiana) del vector que definen en conjunto los valores de Objeto, Propiedad y Relaciónrol.

\begin{tabular}{|c|c|c|l|}
\hline SC & M & Fórmula & \multicolumn{1}{|c|}{ Descripción } \\
\hline 1.1 & M1 & $\mathrm{n}(\mathrm{O})$ & Número de tipos de objetos por tipo de producto de trabajo. \\
\hline 1.1 & $\mathrm{M} 2$ & $\mathrm{n}(\mathrm{P})$ & $\begin{array}{l}\text { Número de tipo de propiedades por tipo de producto de } \\
\text { trabajo }\end{array}$ \\
\hline 1.1 & $\mathrm{M} 3$ & $\mathrm{n}(\mathrm{R})$ & Número de tipos de relaciones por producto de trabajo \\
\hline 1.1 & $\mathrm{M} 6$ & $\mathrm{C}^{\prime}(\mathrm{wp})=\sqrt{n(o)^{2}+n(P)^{+}+n(R)^{2}}$ & Complejidad conceptual por tipo de producto de trabajo. \\
\hline 1.2 & $\mathrm{M} 1$ & $\mathrm{BTI}=\mathrm{NWPin}(\mathrm{T})+\mathrm{NWPout}(\mathrm{T})$ & $\begin{array}{l}\text { Interacción básica de tareas es la suma de la cantidad de } \\
\text { productos de trabajo entrada y la de salida de cada tarea. }\end{array}$ \\
\hline 1.3 & $\mathrm{M} 7$ & $\mathrm{C}^{\prime}(\mathrm{M})=\sum_{w p \in W P} C^{\prime}(w p)$ & $\begin{array}{l}\text { Complejidad total de los productos de trabajo de la } \\
\text { metodología. }\end{array}$ \\
\hline
\end{tabular}

Tabla 1. Métricas de complejidad.

Adaptada de (Perepletchikov et al., 2013) 
Fig. 1: $\quad$ Modelo de calidad para metodologías de desarrollo de software. Adaptado de (Perepletchikov et al., 2013)

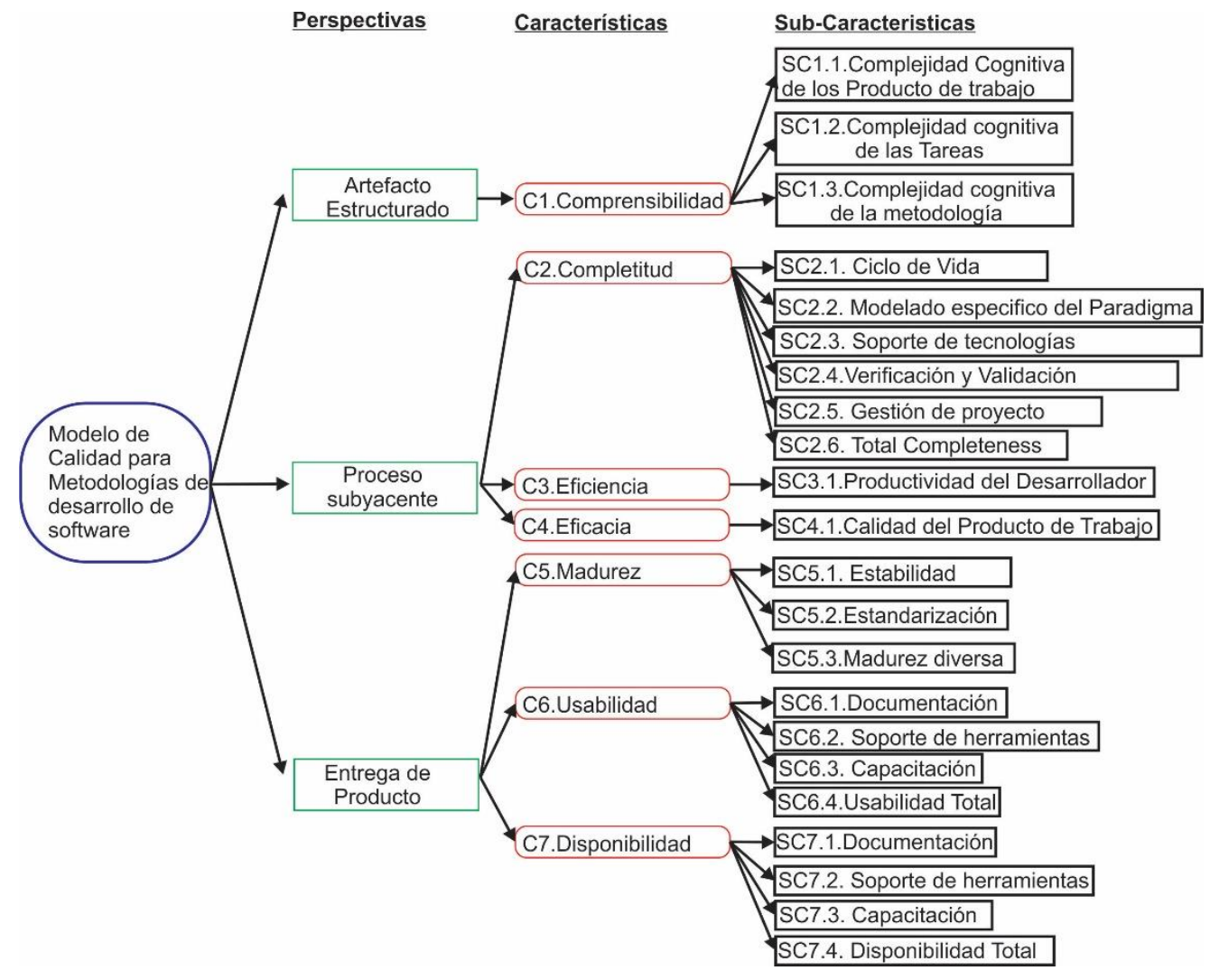

\begin{tabular}{|c|c|l|}
\hline ID & Métrica & \multicolumn{1}{c|}{ Definición } \\
\hline R1 & $\mathrm{n}(\mathrm{OT})$ & $\begin{array}{l}\text { Número de tipos de objetos de la } \\
\text { técnica. }\end{array}$ \\
\hline $\mathrm{R} 2$ & $\mathrm{n}(\mathrm{RT})$ & $\begin{array}{l}\text { Número de conceptos con que se } \\
\text { describen las conexiones entre los } \\
\text { objetos. }\end{array}$ \\
\hline $\mathrm{R} 3$ & $\mathrm{n}(\mathrm{PT})$ & $\begin{array}{l}\text { Número de propiedades existentes } \\
\text { dentro de la técnica que definen al } \\
\text { objeto o la relación. }\end{array}$ \\
\hline $\mathrm{R} 4$ & $\mathrm{C}^{\prime}(\mathrm{M})$ & $\begin{array}{l}\text { Complejidad conceptual cuya } \\
\text { fórmula es: } \\
C^{\prime}(M) \\
=\sqrt{n\left(O_{T}\right)^{2}+n\left(R_{T}\right)^{2}+n\left(P_{T}\right)^{2}}\end{array}$ \\
\hline
\end{tabular}

Tabla 2. Métricas de complejidad.

Adaptada de (Rossi \& Brinkkemper, 1996)

\subsection{Métricas de García y Otros}

En (García et al., 2006), como parte del marco para el modelado y evaluación de procesos software (FMESP, por sus siglas en inglés de Framework for the Modeling and Evaluation of Software Processes), se proponen 12 métricas relacionados al proceso de software. Para la presente investigación se consideraron de (García et al., 2006) las siguientes 4 métricas que se han precedido en su identificación con la letra G, tal como se observa en la Tabla 3. 


\begin{tabular}{|l|l|l|}
\hline Id & \multicolumn{1}{|c|}{ Métrica } & \multicolumn{1}{|c|}{ Definición } \\
\hline G1 & NA & Número de actividades del modelo de proceso software \\
\hline G2 & $\begin{array}{l}\text { NDWPin, de Number of input Dependences } \\
\text { of the Work Products with the Activities in } \\
\text { the Process }\end{array}$ & $\begin{array}{l}\text { Número de entradas dependientes de los productos de } \\
\text { trabajo con las actividades en los procesos }\end{array}$ \\
\hline G3 & $\begin{array}{l}\text { NDWPin, de Number of input Dependences } \\
\text { of the Work Products with the Activities in } \\
\text { the Process }\end{array}$ & $\begin{array}{l}\text { El número de salidas dependientes de los productos de } \\
\text { trabajo con las actividades en los procesos }\end{array}$ \\
\hline G4 & $\begin{array}{l}\text { NDWP, de Number of Dependences } \\
\text { between Work Products and Activities }\end{array}$ & $\begin{array}{l}\text { El número de dependencias entre productos de trabajo y } \\
\text { actividades: } \\
\text { NDWP(PM) = NDWPIn(MP) + NDWPOut(MP) (o G4 } \\
\text { = G3+G2). }\end{array}$ \\
\hline
\end{tabular}

Tabla 3. Métricas para modelos de procesos software

\subsection{Trabajos Relacionados}

El enfoque métrico de complejidad conceptual fue aplicado en varias investigaciones como: en el método de medición de la complejidad del modelo de gestión de casos y notación (Marin, Lotriet, \& Van Der Poll, 2014); en la evaluación de eficiencia de proyectos de desarrollo de software (Cao, Gu, \& Thompson, 2012); en (Erickson \& Siau, 2005) se desarrolla una medición de complejidad de la técnica UML en la práctica; en (Erickson \& Siau, 2004) se destaca la importancia de la complejidad teórica y práctica de un método de desarrollo de sistemas; y, en (Zhang, Kishore, Sharman, \& Ramesh, 2007) se realiza una comparación de complejidades entre varios diagramas de UML y un lenguaje de modelado de integración ágil.

\subsection{Gestión de Proyecto en la ISO/IEC 29110-5-1-2}

La ISO/IEC 29110-5-1-2 (ISO/IEC, 2011) publicada en enero del 2011, es el perfil básico en ingeniería de software y comprende los procesos de Gestión de Proyectos (GP) e Implementación de Software (IS). Estos procesos comprenden prácticas que se basan en la ISO/IEC 12207:2008 (procesos de ciclo de vida del software) y los contenidos de los productos de software desarrollados en la ISO/IEC 15289:2006 pero seleccionadas y adaptadas para pequeñas organizaciones (ISO/IEC, 2011), considerando que una pequeña organización tienen hasta 25 personas en desarrollo de software (ISO/IEC, 2016).

Con respeto al flujo de información del proceso de gestión de proyectos de la ISO/IEC 29110-5-1-2 (ver Figura 2), este contiene entregables de entrada, salida e internos que permiten la interacción entre las 4 actividades (ISO/IEC, 2011): (i) planeación del proyecto; (ii) ejecución del plan de proyecto; (iii) evaluación y control del plan de proyecto; y (iv) cierre del proyecto. Así mismo (ISO/IEC, 2011), cada tarea tiene productos de entradas y productos de salida; los productos de entrada tienen como fuente una entidad o proceso externo; y los productos de salida tienen como destino una entidad o proceso externo. 


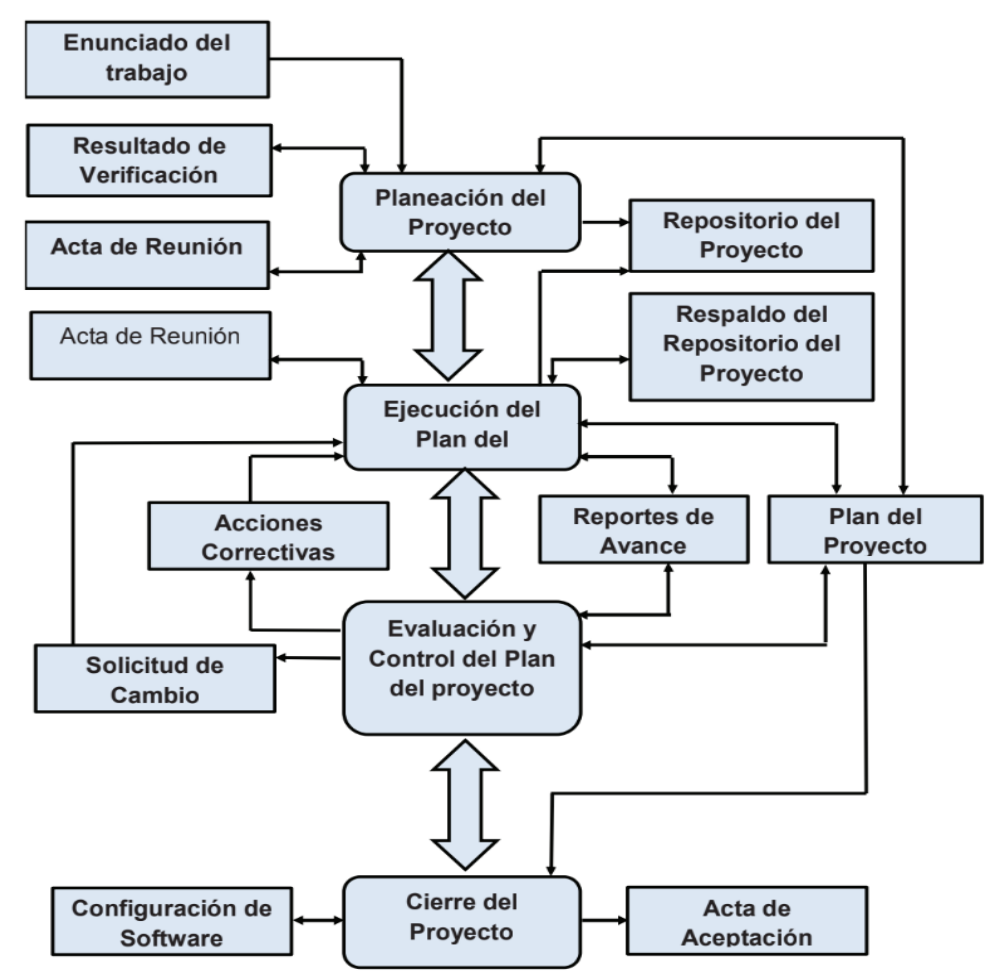

Fig. 2: Proceso de Gestión de Proyectos, Adaptado de (ISO/IEC, 2011)

\subsection{Gestión de Proyecto del PMBOK}

La Guía PMBOK es un conjunto de buenas prácticas reconocidas que son ampliamente aplicadas por profesionales de la gestión de proyectos para la gestión exitosa de proyectos en todo el mundo (Merquida \& Antonia, 2014). Estas buenas prácticas de gestión cubren todo el ciclo de vida de un proyecto (Mas \& Mesquida, 2013). Esta Guía (PMI, 2017) contiene 5 grupos de procesos para la dirección de proyectos: (i) grupo de procesos de inicio; (ii) grupo de proceso de planificación; (iii) grupo de procesos de ejecución; (iv) grupo de procesos de monitoreo y control; y (v) grupo de procesos de cierre; y establece 10 áreas de conocimiento: (i) .integración, (ii) alcance, (iii) cronograma, (iv) costos, (v) calidad, (vi) recursos, (vii) comunicaciones, (viii) riesgos, (ix) adquisiciones, y (x) interesados. La estructura contenido en el PMBOK (PMI, 2017) identifica para cada área de conocimiento un conjunto de procesos relacionados como por ejemplo, para el área de conocimiento 4. Gestión de la Integración del Proyecto, donde se identifica 7 procesos, de los cuales se presentan 3 a continuación: 4.1 Desarrollar el Acta de Constitución del Proyecto; 4.2 Desarrollar el Plan para la Dirección del Proyecto; y 4.3 Dirigir y gestionar el Trabajo del Proyecto. Y por último, cada proceso se caracteriza a través de sus entradas, salidas y en conjunto las herramientas y técnicas. 


\section{MÉTRICAS PARA EL ANÁLISIS COMPARATIVO}

En esta sección se presenta la selección y desarrollo de las métricas para el estudio comparativo. Estas métricas se presentan de manera agrupadas por las sub características tal como se define en el modelo de (Perepletchikov et al., 2013). Las sub características son: (i) complejidad conceptual de las actividades y proceso; (ii) complejidad de las dependencias; y (iii); complejidad cognitiva de la metodología. Para nuestro estudio se ha considerado identificar las métricas definidas para calcular la complejidad cognitiva con el prefijo $Z$ y un correlativo quedando desde Z1 hasta la Z12, los que se presentan a continuación:

a. Complejidad estructural del entregable. Para determinar las métricas a utilizar para esta complejidad, se ha tomado como referencia (Perepletchikov et al., 2013), (Göpferich, 2009) y (Arthur \& Stevens, 1989). La métrica se basa en la descomposición de la comprensibilidad aplicado a un entregable (tipo documento) como: (i) coherencia entre títulos y contenidos, (ii) organización en secuencia lógica dirigida por los títulos, (iii) la amplitud del contenido determinada por la estructura del documento o índice; y, (iv) profundidad del contenido determinada por la estructura del documento (índice). Las métricas de Z1 a Z5 se presentan en la Tabla 4 y se obtienen de un instrumento que incluye las estructuras de los documentos asociados a cada propuesta. Para la calificación se utiliza una escala de Likert.

b. Complejidad conceptual de las actividades y proceso. Para determinar esta complejidad se utilizó como referencia las métricas, criterios y filosofía usados en la definición desarrollada en (Rossi \& Brinkkemper, 1996) y las propuestas de (Perepletchikov et al., 2013) y (García et al., 2006). Las métricas definidas para nuestro estudio se presentan en la Tabla 4 y se describen a continuación: (i) la métrica Z6, considerando lo de (Perepletchikov et al., 2013), se define por analogía a partir de la métrica M1 de la SC1.2 (Ver Tabla 1); (ii) la métrica Z7, considerando lo de (García et al., 2006), se definió a partir de la métrica G1; y la métrica Z8 se obtuvo de las métrica G4 (que incluye dos métricas parciales como se observa en la Sección 2.3; (iii) la métrica Z9, basado en (Rossi \& Brinkkemper, 1996), se obtuvo de la métrica R4 de la Tabla 2. La métrica Z10 que representa la complejidad del proceso se obtienen de la sumatoria de las métricas anteriores y que está relacionada a con la M7 de la SC.1.3 (ver Tabla 1).

c. Complejidad de las dependencias. Dentro de esta complejidad se definió la métrica Z11 que se basó en lo definido en (García et al., 2006) como la métrica de número de dependencias descrito en la Tabla 3.

d. Complejidad cognitiva de la metodología. Para determinar la complejidad cognitiva de la metodología se usó el concepto espacio tridimensional del trabajo de (Rossi \& Brinkkemper, 1996). Para dicho espacio se utilizó de base el Entregable, Proceso y Dependencias siendo las métricas respectivas: (i) $C^{\prime}(E n)$, complejidad estructural del entregable; (ii) $C^{\prime}(P)$, complejidad total del proceso y (iii) $N D(P)$, número de dependencias de los entregables con todas las actividades. En la Tabla 4 se presenta la fórmula establecida para la métrica Z12 que es la complejidad cognitiva de la metodología. 


\section{RESULTADOS}

En esta sección se presenta los resultados de las mediciones de complejidad cognitiva a través de las métricas establecidas, ver Tabla 4, para la ISO/IEC 29110-5-1-2 y una implementación completa del PMBOK.

a. Complejidad Cognitiva en la Gestión de Proyectos de la ISO/IEC 291105-1-2. Se tomó como base la Sección 9 Descripción de Producto del documento de la ISO (ISO/IEC, 2011).

Las métricas de complejidad estructural del entregable obtenidas fueron. Z1 $=64, Z 2=64, Z 3=64, Z 4=64$, y a partir de ello se obtiene $Z 5=64$ Estas métricas a través de una evaluación por parte de expertos temáticos y usuarios avanzados.

Las métricas de complejidad conceptual total por actividades y de proceso obtenidas se presentan en la Tabla 5 , donde se calcula por cada actividad las métricas Z6, Z7, Z8 y Z9. Siendo Z9 obtenida en función a Z6, Z7 y Z8. El valor de $Z 10$ se obtiene de la suma de los valores parciales de Z9, lo que da un total de $Z 10=78.7$.

La métrica de dependencias por proceso, identificado como Z11, se calcula analizando entradas y salidas en la Figura 2, obteniendo el siguiente resultado: $Z 11=14$ entradas +13 salidas $=27$. 


\begin{tabular}{|c|c|c|}
\hline $\mathbf{M}$ & Definición & Descripción \\
\hline $\mathrm{Z} 1$ & $\begin{array}{l}\text { Coherencia de títulos con } \\
\text { contenidos. }\end{array}$ & $\begin{array}{l}\text { Esta métrica mide la característica de los títulos adecuados en función del } \\
\text { contenido del documento. ¿Los títulos de las secciones representan } \\
\text { adecuadamente los contenidos del tipo de documento? }\end{array}$ \\
\hline $\mathrm{Z} 2$ & $\begin{array}{l}\text { Organización en } \\
\text { secuencia lógica dirigida } \\
\text { por los títulos. }\end{array}$ & $\begin{array}{l}\text { Esta métrica mide la característica de los títulos adecuados en función de la } \\
\text { secuencia lógica del contenido del documento. ¿Los títulos de las secciones } \\
\text { siguen un orden (transición temática o secuencia lógica) adecuado para el } \\
\text { tipo de documento? }\end{array}$ \\
\hline $\mathrm{Z} 3$ & $\begin{array}{l}\text { Amplitud del contenido } \\
\text { determinada por la } \\
\text { estructura del documento } \\
\text { (índice). }\end{array}$ & $\begin{array}{l}\text { Esta métrica mide la estructura del ¿La estructura del tipo de documento } \\
\text { (en número de secciones en cada nivel) organiza de manera adecuada el } \\
\text { contenido? }\end{array}$ \\
\hline $\mathrm{Z} 4$ & $\begin{array}{l}\text { Profundidad del contenido } \\
\text { determinada por la } \\
\text { estructura del documento } \\
\text { (índice). }\end{array}$ & $\begin{array}{l}\text { ¿La estructura del tipo de documento (en profundidad de niveles) organiza } \\
\text { de manera adecuada el contenido? }\end{array}$ \\
\hline $\mathrm{Z} 5$ & $C^{\prime}(E n)$ & $\begin{array}{l}\text { Complejidad estructural del entregable } \\
\qquad \mathrm{Z} 5=(\mathrm{Z} 1+\mathrm{Z} 2+\mathrm{Z} 3+\mathrm{Z} 4) / 4\end{array}$ \\
\hline Z6 & NEn(A) & $\begin{array}{l}\text { Número de entregables totales por actividad. } \\
\text { NEn(A)=NEnin }(A)+\text { NEnout }(A) \\
\text { Donde } \\
\text { NEnin = número de entregables de entrada } \\
\text { NEnout = número de entregables de salida } \\
\text { A = actividad }\end{array}$ \\
\hline $\mathrm{Z7}$ & NT(A) & Número de tareas por actividad. \\
\hline $\mathrm{Z} 8$ & NDEnT(A) & $\begin{array}{l}\text { Número total de dependencias de los entregables con todas las tareas por } \\
\text { actividad. } \\
\text { NDEnT(A)= NDEnTin(A) + NDEnTout(A) } \\
\text { Donde } \\
\text { NDEnTin(A) = número de dependencias de entrada del entregable con } \\
\text { todas las tareas. } \\
\text { NDEnTout(A) = número de dependencias de salida del entregable con todas } \\
\text { las tareas. } \\
\text { A = actividad }\end{array}$ \\
\hline $\mathrm{Z9}$ & $\mathrm{C}^{\prime}(\mathrm{A})$ & $\begin{array}{l}\text { Complejidad conceptual total por actividad. } \\
\qquad \begin{array}{l}C^{\prime}(A)=\sqrt{N E n(A)^{2}+N T(A)^{2}+N D E n T(A)^{2}} \\
Z 9=\sqrt{(Z 6)^{2}+(Z 7)^{2}+(Z 8)^{2}}\end{array}\end{array}$ \\
\hline $\mathrm{Z} 10$ & $\mathrm{C}^{\prime}(\mathrm{P})$ & $\begin{array}{l}\text { Complejidad total por proceso } \\
\qquad \begin{array}{c}\mathrm{C}^{\prime}(\mathrm{P})=\sum C^{\prime}(A) . .(\mathrm{ver} \mathrm{Z} 9) \\
\mathrm{Z} 10=\sum(Z 9)\end{array}\end{array}$ \\
\hline $\mathrm{Z} 11$ & $\mathrm{ND}(\mathrm{P})$ & $\begin{array}{l}\text { Número de dependencias por proceso } \\
\text { ND }(\mathrm{P})=\text { NDEnAin }(\mathrm{P})+\mathrm{NDEnAout}(\mathrm{P}) \\
\text { Donde } \\
\text { NDPEnAin = número de dependencias de entrada de los entregables con } \\
\text { todas las actividades. } \\
\text { NDPEnAout = número de dependencias de salida de los entregables con } \\
\text { todas las actividades. }\end{array}$ \\
\hline $\mathrm{Z} 12$ & $\mathrm{C}^{\prime}(\mathrm{M})$ & $\begin{array}{l}\text { Complejidad cognitiva de la metodología } \\
\qquad \begin{array}{r}C^{\prime}(M)=\sqrt{C^{\prime}(E n)^{2}+C^{\prime}(P)^{2}+N D(P)^{2}} \\
Z 12=\sqrt{(Z 5)^{2}+(Z 10)^{2}+(Z 11)^{2}}\end{array}\end{array}$ \\
\hline
\end{tabular}

Tabla 4. Métricas para evaluación de la complejidad cognitiva 
Finalmente, la complejidad cognitiva de la ISO/IEC 29110-5-1-2, se establece en $\mathrm{Z} 12=104.97$.

\begin{tabular}{|l|l|l|l|l|}
\hline Actividad & $\mathbf{Z 6}$ & $\mathbf{Z 7}$ & $\mathbf{Z 8}$ & $\mathbf{Z 9}$ \\
\hline PM.1 Planificacion de proyecto & 5 & 15 & 35 & 38.4 \\
\hline PM.2 Ejecucion del plan de proyecto & 7 & 6 & 22 & 23.9 \\
\hline PM.3 Eval. y control del plan de proyecto & 4 & 3 & 7 & 8.6 \\
\hline PM.4 Cierre de proyecto & 3 & 2 & 7 & 7.9 \\
\hline
\end{tabular}

Tabla 5. Resultados de la complejidad conceptual de cada actividad en Gestión de Proyecto de la ISO/IEC 29110-5-1-2

b. Complejidad cognitiva de una implementación completa PMBOK. Se tomó como base una implementación desarrollada por una empresa consultora de dirección de proyectos en el sector de tecnologías de información. Por acuerdos de confidencialidad el nombre de la empresa se mantendrá en reserva. Considerando la manera en que trabaja la Consultora, se realizó el análisis por grupos de procesos del PMBOK.

Las métricas de complejidad estructural del entregable obtenidas fueron. Z1 $=90, Z 2=90, Z 3=90, Z 4=89$, y a partir de ello se obtiene $Z 5=89.75$.

Las métricas de complejidad conceptual total por actividades y de proceso obtenidas se presentan en la Tabla 6 , donde se calcula por cada actividad las métricas Z6, Z7, Z8 y Z9. El valor de Z10 se obtiene de la suma de los valores parciales de $Z 9$, lo que da un total de $Z 10=420.9$.

La métrica de dependencias por proceso, es decir $Z 11=99$, esta se obtiene del conteo realizado en el gráfico de interacciones entre procesos de la dirección de proyectos de la tercera sección página 53 del PMBOK.

Finalmente, la complejidad cognitiva del PMBOK, se determina según la métrica Z12 igual a 441.6 .

\begin{tabular}{|l|c|c|c|c|}
\hline Grupo de procesos de la dirección de proyecto & $\mathbf{Z 6}$ & $\mathbf{Z 7}$ & $\mathbf{Z 8}$ & $\mathbf{Z 9}$ \\
\hline Grupo de procesos de Inicio & 3 & 2 & 11 & 11.6 \\
\hline Grupo de procesos de planificación. & 39 & 24 & 202 & 207.1 \\
\hline Grupo de procesos de ejecución. & 9 & 8 & 71 & 72.0 \\
\hline Grupo de procesos de monitoreo y control. & 5 & 11 & 116 & 116.6 \\
\hline Grupo de procesos de cierre & 10 & 2 & 9 & 13.6 \\
\hline
\end{tabular}

Tabla 6. Resultados de la complejidad conceptual del grupo de proceso del PMBOK 


\section{DISCUSIÓN Y TRABAJO FUTURO}

En este artículo se obtuvieron los resultados de un análisis de complejidad cognitiva, determinándose que el proceso de gestión de proyectos de la ISO/IEC 29110-5-1-2 obtuvo una complejidad cognitiva de 104.97 y para el caso de la implementación basada en el PMBOK se obtuvo una complejidad cognitiva de 441.6. Estos resultados evidencian que la ISO/IEC 29110-5-1-2 es menos compleja en comparación con la implementación completa basada PMBOK, e implica además que el esfuerzo de implementar un modelo basado en la ISO/IEC 29110-5-1-2 es menor que una implementación completa basada en el PMBOK.

De otro lado, para el caso de definir una metodología basada en PMBOK, se puede utilizar la complejidad cognitiva para evaluar su facilidad y esfuerzo de adopción en una organización.

Además, esta investigación propone métricas para medir la complejidad estructural de los entregables, traduciéndose como la complejidad cognitiva. Como trabajo futuro se propone realizar una medición de la complejidad cognitiva de los procesos de desarrollo de software de las pequeñas empresas en comparación con la ISO/IEC 12207. Asimismo resulta interesante contar con una herramienta de software para automatizar el cálculo de estas métricas de complejidad para la comparación de modelos.

\section{AGRADECIMIENTOS}

Este trabajo ha sido desarrollado gracias al apoyo brindado por el Departamento de Ingeniería de la Pontificia Universidad Católica del Perú, en el marco de la movilidad académica de la Red Peruana de Universidades. 


\section{REFERENCIAS}

Arthur, J. D., \& Stevens, K. T. (1989). Assessing the adequacy of documentation through document quality indicators. Conference on Software Maintenance, 40-49.

Cao, Q., Gu, V. C., \& Thompson, M. A. (2012). Using complexity measures to evaluate software development projects: A nonparametric approach. Engineering Economist, 57(4), 274-283. https://doi.org/10.1080/0013791X.2012.729878

Erickson, J., \& Siau, K. (2004). Theoretical and practical complexity of modeling methods. Communications of the ACM, 50(8), 46-51. https://doi.org/10.1145/1278201.1278205

Erickson, J., \& Siau, K. (2005). Toward Practical Measures of Complexity in Real Time Modeling Methods. 11th Americas Conference on Information Systems, 3067-3074.

García, F., Piattini, M., Ruiz, F., Canfora, G., \& Visaggio, C. A. (2006). FMESP: Framework for the modeling and evaluation of software processes. Journal of Systems Architecture, 52(11), 627-639. https://doi.org/10.1016/j.sysarc.2006.06.007

Göpferich, S. (2009). Comprehensibility assessment using the Karlsruhe Comprehensibility Concept. The Journal of Specialised Translation, (11), 12-38. Retrieved from http://www.jostrans.org/issue11/art_goepferich.pdf

ISO/IEC. (2011). ISO/IEC TR 29110-5-1-2:2011 Software Engineering -Lifecycle Profiles for Very Small Entities (VSEs) -- Part 5-1-2: Management and Engineering Guide: Generic Profile Group: Basic Profile. Retrieved from https://www.iso.org/standard/51153.html

ISO/IEC. (2016). ISO/IEC TR 29110-1:2016 Systems and software engineering -- Lifecycle profiles for Very Small Entities (VSEs) -- Part 1: Overview. Retrieved from https://www.iso.org/standard/62711.html

Marin, M. A., Lotriet, H., \& Van Der Poll, J. A. (2014). Measuring Method Complexity of the Case Management Modeling and Notation (CMMN). 209-216. https://doi.org/10.1145/2664591.2664608

Mas, A., \& Mesquida, A. L. (2013). Software project management in small and very small entities | Gestión de proyectos de software en pequeñas y micro empresas. Iberian Conference on Information Systems and Technologies, CISTI, 406-412.

Merquida, A.-L., \& Antonia, M. (2014). A project management improvement program according to ISO/IEC 29110 and PMBOK. Journal of Software: Evolution and Process. https://doi.org/10.1002/smr.1665

Mitani, Y., Matsumura, T., Barker, M., Tsuruho, S., Inoue, K., \& Matsumoto, K. (2007). Proposal of a Complete Life Cycle In-Process Measurement Model Based on Evaluation of an In-Process Measurement Experiment Using a Standardized Requirement Definition Process. https://doi.org/10.1109/ESEM.2007.27

Oliveira, J. M. M. De, Oliveira, K. B. De, \& Belchior, A. D. (2006). Measurement Process : A Mapping Among CMMI-SW, ISO / IEC 15939, IEEE Std 1061 , Six Sigma and PSM. 15939(2002).

Perepletchikov, M., Ryan, C., \& Tari, Z. (2013). An Analytical Framework for Evaluating Service-Oriented Software Development Methodologies. https://doi.org/10.4304/jsw.8.7.1642-1659 
PMI. (2017). Guía de los Fundamentos para la Dirección de Proyectos.

Ram, P., Rodriguez, P., \& Oivo, M. (2018). Software Process Measurement and Related Challenges in Agile Software Development : A Multiple Case Study.

Rossi, M., \& Brinkkemper, S. (1996). Complexity metrics for systems development methods and techniques. Information Systems, 21(2), 209227. https://doi.org/10.1016/0306-4379(96)00012-9

Slavek, N., Lukić, I., \& Köhler, M. (2012). Software process measuring model [Model mjerenja softverskog procesa]. Tehnicki Vjesnik, 19(1), 11-17. Retrieved from http://www.scopus.com/inward/record.url?eid=2-s2.084859181540\&partnerID=40\&md5=870159bb67251643fecf0dab02b9beb5

Zhang, H., Kishore, R., Sharman, R., \& Ramesh, R. (2007). Agile Integration Modeling Language (AIML): A conceptual modeling grammar for agile integrative business information systems. Decision Support Systems, 44(1), 266-284. https://doi.org/10.1016/j.dss.2007.04.009

\section{NOTAS BIOGRÁFICAS}

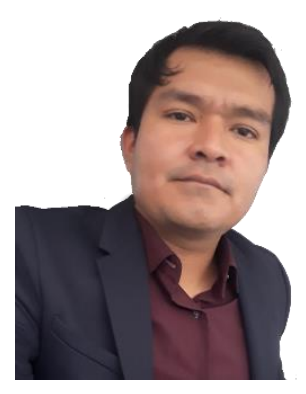

Edinson Damian es Ingeniero de Sistemas de la Universidad Nacional Pedro Ruiz Gallo, y actualmente, egresado de Maestría con Mención en Gerencia de TI y Gestión de Software en la escuela de Posgrado de la Universidad Nacional Pedro Ruiz Gallo. Su área de investigación es Medición del proceso software. Se ha desempeñado como analista de sistemas y consultor en el sector privado de Perú.

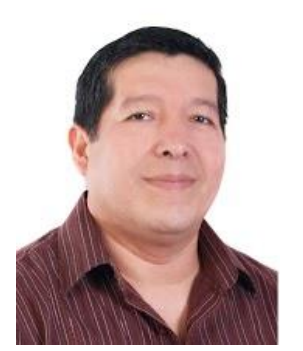

Abraham Dávila es investigador y profesor principal en la Pontificia Universidad Católica del Perú (PUCP) desde el 2000. Actualmente es director e investigador principal del proyecto ProCalProSer (2013-2016 Fase I y 2017-2019 Fase II) y director y miembro fundador de GIDIS-PUCP. Posee el grado de bachiller en ciencias con mención en Ingeniería Mecánica y magister en Informática por la PUCP. Miembro del grupo de trabajo de la ISO/IEC que elabora la serie de normas ISO/IEC 29110. Sus principales áreas de interés son: calidad en informática (a nivel de proceso software, producto software y gestión de servicios) y educación en ingeniería de software. 


\section{APÉNDICE A: DETERMINACIÓN DE MÉTRICAS}

Ejemplo para determinar la complejidad conceptual en la ISO/IEC 29110-51-2

La métrica Z6 se obtiene por un conteo de los entregables que entran y salen (Ver Figura 2). Esos entregables son: plan de proyecto, configuración de software y acta de aceptación y por tanto el valor de Z6 = 3 .

La métrica Z7 se obtiene contando la cantidad de tareas por actividad. Así, en la Tabla A.1, se observa que la actividad PM.4 referida a cierre de proyectos que tiene dos tareas: PM.4.1 y PM.4.2; por lo que $Z 7=2$.

La métrica Z8 se obtiene contando la cantidad de dependencias de entregables, esto es la suma de los entregables que entran y los entregables que salen. Esto significa que para ambas tareas PM.4.1 y PM.4.2 se tiene un total de 4 dependencias a la entrada y 3 dependencias a la salida, lo que da un total de $\mathrm{Z} 8=7$ dependencias.

\begin{tabular}{|c|c|c|}
\hline Lista de tareas & $\begin{array}{l}\text { Entrada de } \\
\text { productos }\end{array}$ & $\begin{array}{l}\text { Salida de } \\
\text { productos }\end{array}$ \\
\hline $\begin{array}{l}\text { PM.4.1 Formalizar la } \\
\text { terminación del proyecto de } \\
\text { acuerdo con las instrucción de } \\
\text { entrega establecidas en el plan } \\
\text { de proyecto, proporcionar } \\
\text { apoyo de aceptación y obtener } \\
\text { el registro de aceptación } \\
\text { firmado }\end{array}$ & $\begin{array}{l}\text { Plan de } \\
\text { proyecto } \\
\text { - instrucciones } \\
\text { de entrega } \\
\text { Configuración } \\
\text { de software } \\
\text { (entregable) }\end{array}$ & $\begin{array}{l}\text { Acta de } \\
\text { aceptación } \\
\text { Configuración } \\
\text { de software } \\
\text { (Aceptado) }\end{array}$ \\
\hline $\begin{array}{l}\text { PM. } 4.2 \text { Actualizacion del } \\
\text { respositorio de proyecto }\end{array}$ & 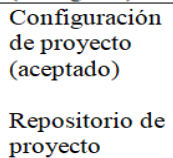 & $\begin{array}{l}\text { Repositorio de } \\
\text { proyecto } \\
\text { (actualizado) }\end{array}$ \\
\hline
\end{tabular}

Tabla A.1. Lista de tareas del PM.4 cierre de proyecto de la ISO/IEC 29110-5$1-2$

\section{Ejemplo para determinar la complejidad conceptual del PMBOK}

La métrica Z6 se obtiene por un conteo de los entregables del grupo de inicio que entran y salen. Estos entregables son: Acta de constitución del proyecto, Registro y clasificación de los interesados, Checklist de presentación para reunión de inicio de proyecto, por tanto el valor de $Z 6=3$.

La métrica $\mathrm{Z7}$ se obtiene realizando un conteo de la cantidad de actividades del área de conocimientos pertenecientes al grupo de procesos de inicio, es decir Gestión de la integración del proyecto y Gestión de interesados del proyecto. Así, en la Tabla A.2, se observa que el grupo de procesos de inicio tiene dos áreas de conocimiento; por lo que $Z 7=2$.

La métrica Z8 se obtiene contando la cantidad de dependencias de entregables, esto es la suma de los entregables que entran y los entregables que salen. Esto significa que para ambas áreas de conocimiento cuatro y trece se tiene un total de 6 dependencias a la entrada y 5 dependencias a la salida, lo que da un total de $Z 8=11$ dependencias. 


\begin{tabular}{|l|l|l|l|}
\hline $\begin{array}{l}\text { Área de } \\
\text { conocimiento }\end{array}$ & $\begin{array}{l}\text { Grupo de } \\
\text { procesos de } \\
\text { inicio }\end{array}$ & Productos de entrada & $\begin{array}{l}\text { Productos } \\
\text { de salida }\end{array}$ \\
\hline $\begin{array}{l}\text { 4. Gestión de } \\
\text { integración del } \\
\text { proyecto }\end{array}$ & $\begin{array}{l}4.1 \\
\text { Desarrollar el } \\
\text { acta de } \\
\text { constitución } \\
\text { de proyecto. }\end{array}$ & $\begin{array}{l}\text {-Enunciado de trabajo } \\
\text {-Caso de negocio } \\
\text {-Acuerdos } \\
\text {-Factores ambientales } \\
\text { de la empresa. } \\
\text {-Activos de los } \\
\text { procesos la } \\
\text { organización }\end{array}$ & $\begin{array}{l}\text {-Acta de } \\
\text { constitució } \\
\text { n de } \\
\text { proyecto }\end{array}$ \\
\hline $\begin{array}{l}\text { 13. Gestión de } \\
\text { los interesados } \\
\text { del prouecto }\end{array}$ & $\begin{array}{l}\text { Identificar a } \\
\text { los } \\
\text { interesados }\end{array}$ & $\begin{array}{l}\text {-Documento de } \\
\text { adquisición. } \\
\text {-Factores ambientales } \\
\text { de la empresa } \\
\text {-Activos de los } \\
\text { procesos de la } \\
\text { organización }\end{array}$ & $\begin{array}{l}\text {-Registro } \\
\text { interesados. }\end{array}$ \\
\hline
\end{tabular}

Tabla A.2. Grupo de Procesos de Inicio del PMBOK.

Esta obra está bajo una licencia de Creative Commons Reconocimiento-NoComercial-Compartirlgual 2.5 México. 